\title{
Polyamine Transport in Aspergillus nidulans
}

\author{
By D. H. SPATHAS, $\dagger$ J. A. PATEMAN $\ddagger$ And A. J. ClutTERBUCK* \\ Department of Genetics, University of Glasgow, Glasgow G11 5JS, U.K.
}

(Received 2 February 1981; revised 15 July 1981)

\begin{abstract}
The uptake of putrescine, spermidine and spermine was studied in Aspergillus nidulans using ${ }^{14} \mathrm{C}$-labelled polyamines. Active transport systems, inhibited by azide and regulated by nitrogen availability, exist at least for putrescine and spermidine. Putrescine is taken up two to three times more rapidly than spermidine, reflecting a lower $K_{\mathrm{m}}$ for the former substrate. The two uptake systems appear to be independent, spermidine uptake being inhibited by both putrescine and spermine, while putrescine uptake is not inhibited by the other two polyamines. The relationships of these transport systems to the tenfold or greater difference between spermidine and putrescine concentrations required to support growth of the putrescine auxotroph are discussed.
\end{abstract}

\section{INTRODUCTION}

Polyamines are ubiquitous constituents of both eukaryotes and prokaryotes, although quantitative and qualitative variations exist between organisms even of the same class (Bachrach, 1973). Their precise function is not known, but their participation in a wide variety of physiological processes, as well as the existence of polyamine auxotrophs in Aspergillus nidulans (Sneath, 1955) and other organisms (for reviews, see Tabor \& Tabor, 1976; Stevens \& Winther, 1979), indicate their vital importance.

The existence of a putrescine auxotroph requires the presence of an uptake system at least for putrescine. The $A$. nidulans auxotroph can grow on spermidine in place of putrescine, but only at higher concentrations, an observation that was attributed by Hope \& Stevens (1976) to uptake differences. However, the observed higher accumulation of ${ }^{14} \mathrm{C}$ from labelled putrescine over a period of $9 \mathrm{~h}$ (Hope \& Stevens, 1976) could alternatively be explained in terms of faster metabolic processes for putrescine than for spermidine.

In order to investigate these possibilities, the following studies of short-term polyamine transport systems in $A$. nidulans were undertaken.

\section{METHODS}

Chemicals. All chemicals were obtained from commercial sources. Hydrochloride salts of putrescine, spermidine and spermine were obtained from Sigma. Hydrochloride salts of ${ }^{14} \mathrm{C}$-labelled putrescine, spermidine and spermine were obtained from Amersham. Dansyl chloride was from BDH. Sterile or freshly made solutions were used in all tests.

Media. Media used were essentially those described by Pontecorvo et al. (1953). Nitrogenless minimal medium (NLM) was as described by Cove (1966); this contained $1 \%(w / v)$ glucose which was omitted where specified. Biotin $\left(0.02 \mu \mathrm{g} \mathrm{m}^{-1}\right)$ was added to all growth media, putrescine $(0.06 \mathrm{~mm})$ was added for growth of the putrescine auxotroph, and other supplements were added as described in the text.

Strains of Aspergillus nidulans. The biotin-requiring strain biA1 (Glasgow no. G051) was used as the wild-type with respect to polyamine metabolism. Strain biA1; puA2 is a translocation-free putrescine auxotroph carrying the puA2 mutation which is allelic to puA1 (C. Herman, personal communication; Sneath, 1955). Strain biA1;

$\dagger$ Present address: Department of Biology, University of Patras Medical School, Patras, Greece.

¥ Present address: Department of Genetics, Research School of Biological Sciences, Australian National University, Box 475, Canberra, A.C.T. 2601, Australia. 
are $A^{r} 550$ contains a fully repressing allele at the ammonium regulatory locus areA (Pateman \& Kinghorn, 1976, 1977).

Uptake assays. The mycelium was grown in shaken cultures (1 litre Erlenmeyer flasks containing $200 \mathrm{ml}$ NLM) for $17 \mathrm{~h}$ at $30^{\circ} \mathrm{C}$ with $10 \mathrm{mM}-\mathrm{NaNO}_{3}$ as the sole nitrogen source unless otherwise stated. Mycelium was harvested on a large sintered-glass filter and washed with NLM at $30^{\circ} \mathrm{C}$. It was then resuspended in fresh NLM, without added nitrogen source unless specified, and incubated at $30^{\circ} \mathrm{C}$ on a shaker for another $3 \mathrm{~h}$. Mycelium grown in the form of small pellets under these conditions was harvested, washed again, and pressed between paper towels. A $1.5 \mathrm{~g}$ pressed wet wt portion of mycelium from each flask was divided into three equal parts and immediately resuspended in the uptake flasks containing $50 \mathrm{ml} \mathrm{NLM}$ (supplemented as in the pre-treatment) plus ${ }^{14} \mathrm{C}$-labelled putrescine, spermidine or spermine $\left.[40000 \text { c.p.m. (ml medium) })^{-1}\right]$ and incubated at $25^{\circ} \mathrm{C}$ in a shaker waterbath. The $\mathrm{pH}$ of the uptake medium was $6 \cdot 5$. The concentration of unlabelled polyamine in the flasks was $3 \mathrm{~mm}$ for all three polyamines unless otherwise stated. Samples $(5 \mathrm{ml})$ were taken at $1 \mathrm{~min}$ intervals for the first $5 \mathrm{~min}$. It was found necessary to wash the cells, after harvesting, with $0.05 \mathrm{M}-\mathrm{HCl}$ to remove adhered polyamine (see Results). The radioactivity of the cells was counted in NE250 scintillation fluid (Nuclear Enterprises) with an Intertechnique scintillation counter, and the results were converted to nmol polyamine taken up (mg wet wt) ${ }^{-1}(8 \mathrm{mg}$ wet wt in this system is equivalent to approximately $1 \mathrm{mg}$ dry $\mathrm{wt}$ ).

In the competition experiments, mycelium grown as above was divided into pieces of approximately $80 \mathrm{mg}$ wet wt (weighed accurately after uptake), each of which was suspended in $10 \mathrm{ml}$ NLM uptake medium. Two flasks for each set of uptake conditions were harvested and washed immediately after addition of the labelled polyamine and the resulting c.p.m. (mg wet wt) ${ }^{-1}$ for these unincubated flasks was subtracted from values for the corresponding uptake flasks incubated for $5 \mathrm{~min}$ at $25^{\circ} \mathrm{C}$.

Intracellular accumulation of ${ }^{14} \mathrm{C}$-labelled polyamines. The uptake experiment in this case was allowed to proceed uninterrupted for $30 \mathrm{~min}$ with only radioactive polyamine present. The whole mycelium was then collected, washed with $0.05 \mathrm{M}-\mathrm{HCl}$ and the radioactivity incorporated was determined. Alternatively, cells were freeze-dried after harvesting, extracted, and dansylated for separation and detection of polyamines on silica gel thin-layer chromatography plates (Dion \& Herbst 1970; Spathas, 1978). Quantitative measurements were made by extracting the fluorescent polyamine spots in scintillation fluid and counting the radioactivity as above.

\section{RESULTS}

\section{Adsorption}

Initial experiments suggested that all three polyamines bound strongly to $A$. nidulans walls, as they do to bacteria (Tabor \& Tabor, 1966), and gave an adsorbed fraction which shifted the active uptake when plotting activities against time. This fraction was not washed out by water; however, $\mathrm{HCl}$ at a concentration of $0.05 \mathrm{M}$ was found to be sufficient to clean the cells without affecting the active uptake. Figure 1 shows the effect of washing on putrescine uptake: similar curves were obtained for spermidine and spermine. As a consequence of these observations, cells were always washed with $0.05 \mathrm{M}-\mathrm{HCl}$ after harvesting and the results presented here deal with the active transport only.

In the uptake competition experiments (see below), where higher specific activities accompanied relatively low uptakes, it was found that up to $20 \%$ of the counts represented adsorbed material, even after $\mathrm{HCl}$ washing. Values for unincubated controls were therefore subtracted from the 5 min uptake values in this experiment.

\section{Intracellular accumulation of ${ }^{14} \mathrm{C}$-labelled polyamines}

Comparison of the radioactivity taken up into cells with that left in the medium after 30 min uptake from medium containing very low concentrations of one of the ${ }^{14} \mathrm{C}$-labelled polyamines without carrier, showed that the mycelium could concentrate putrescine 480 -fold, spermidine 80 -fold, but spermine only $1 \cdot 7$-fold under these conditions. Clearly, there are active uptake systems for the first two polyamines, but there is considerable doubt about the third.

In order to see whether the polyamines taken up over a $30 \mathrm{~min}$ period were further metabolized, the cells were extracted and the radioactivity in the separated polyamine spots on thin-layer chromatography plates was measured. After $\left[{ }^{14} \mathrm{C}\right]$ putrescine uptake, $27 \%$ of the counts recovered were found in the putrescine spot while $34 \%$ were in the spot corresponding 


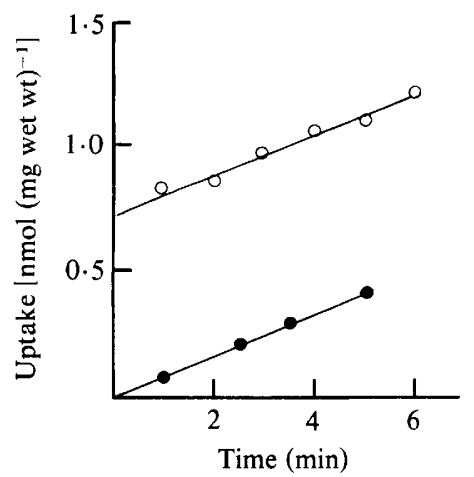

Fig. 1. Uptake of $\left[{ }^{14} \mathrm{C}\right]$ putrescine from a $0.2 \mathrm{~mm}$ solution by wild-type (biAl) mycelium of $A$. nidulans after treatment for $3 \mathrm{~h}$ in nitrogen-free medium: $O$, mycelium washed with distilled water; $O$, mycelium washed with $0.05 \mathrm{M}-\mathrm{HCl}$.
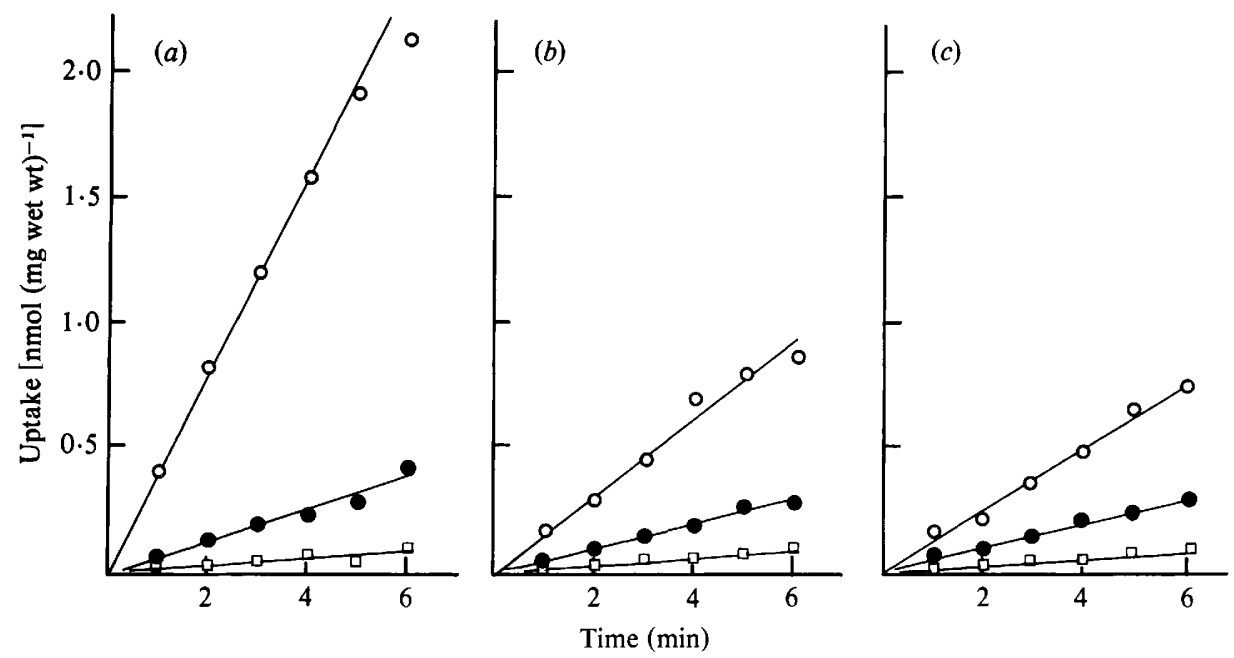

Fig. 2. Uptake of ${ }^{14} \mathrm{C}$-labelled putrescine $(a)$, spermidine $(b)$ and spermine $(c)$ from 3 mm solutions by wild-type (biAl) mycelium of $A$. nidulans after treatment for $3 \mathrm{~h}$ in various media: 0 , nitrogen-free medium; , nitrogen-free medium plus $10 \mu \mathrm{g}$ cycloheximide $\mathrm{ml}^{-1} ; \square$, nitrogen-free medium, but with $1 \mathrm{~mm}$-sodium azide in the uptake medium.

to spermidine; this clearly indicates the conversion of putrescine to spermidine. The extracted materials taken up as spermidine and spermine remained on their own characteristic spots, with the exception of a proportion which, as with putrescine, remained at the chromatographic origin.

\section{Effects of azide and cycloheximide}

The experimental system used gave linear uptake for the first 5-6 min after addition of the mycelium to the uptake flasks (Figs 1 and 2). The respiratory inhibitor sodium azide, at $1 \mathrm{~mm}$ in the uptake medium, effectively inhibited uptake of all three polyamines (Fig. 2), indicating that energy is required for the transport process.

To test the effect of cycloheximide, wild-type cells grown on nitrate for $17 \mathrm{~h}$ were transferred for $3 \mathrm{~h}$ to nitrogen-free medium plus $10 \mu \mathrm{g}$ cycloheximide $\mathrm{ml}^{-1}$ before measurement of uptake. Uptake rates were a third or less of the control rate without 
Table 1. Polyamine transport kinetics for the wild-type (biA1) strain of A. nidulans

The $K_{\mathrm{m}}$ values for polyamine uptake are expressed in $\mathrm{mm}$, and the $V_{\max }$ values in $\mathrm{nmol} \mathrm{min}^{-1}$ (mg wet wt $)^{-1}$.
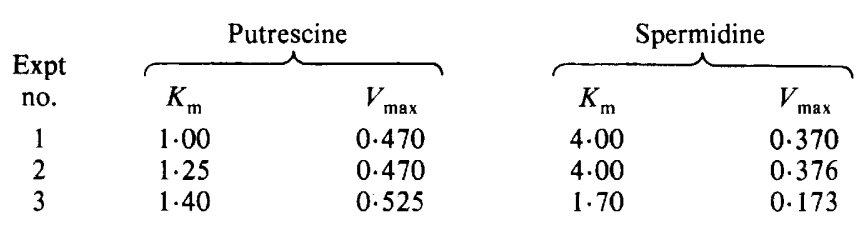

\begin{tabular}{|c|c|}
\hline \multicolumn{2}{|c|}{ Spermine } \\
\hline$K_{\mathrm{m}}$ & $V_{\max }$ \\
\hline $1 \cdot 30$ & 0.148 \\
\hline 0.70 & 0.098 \\
\hline $1 \cdot 10$ & 0.154 \\
\hline
\end{tabular}

Table 2. Inhibition of putrescine and spermidine uptake by other polyamines

Mycelium of the wild-type (biAl) strain was incubated for $5 \mathrm{~min}$ in uptake medium containing $0.5 \mu \mathrm{Ci}$ of labelled polyamine plus unlabelled competitor polyamine. The results shown are the means and standard errors of three determinations [except * which is the mean of two determinations, the third being clearly aberrant (3579 c.p.m. $\left.\mathrm{mg}^{-1}\right)$ ].

\begin{tabular}{|c|c|c|c|}
\hline \multirow[b]{3}{*}{$\begin{array}{l}\text { Unlabelled competitor } \\
\text { polyamine }(4 \mathrm{mM})\end{array}$} & \multicolumn{3}{|c|}{ Uptake [c.p.m. (mg wet wt) $\left.{ }^{-1}\right]$} \\
\hline & \multicolumn{3}{|c|}{${ }^{14} \mathrm{C}$-labelled polyamine $(0.1 \mathrm{~mm}):$} \\
\hline & $\begin{array}{l}\text { Putrescine } \\
\text { (expt 1) }\end{array}$ & $\begin{array}{l}\text { Putrescine } \\
\text { (expt 2) }\end{array}$ & Spermidine \\
\hline None & $322 \pm 38$ & $484 \pm 24$ & $55 \cdot 8 \pm 3.8$ \\
\hline Putrescine & $43 \pm 5$ & - & $14 \cdot 5 \pm 1 \cdot 0$ \\
\hline Spermidine & $320^{*}$ & $461 \pm 11$ & $10.7 \pm 0.7$ \\
\hline Spermine & $349 \pm 29$ & $\div$ & $7 \cdot 4 \pm 0.3$ \\
\hline
\end{tabular}

cycloheximide (Fig. 2). Cycloheximide is an effective protein synthesis inhibitor in $A$. nidulans and therefore it may tentatively be concluded that protein synthesis is necessary for the development of high polyamine transport activities (but see Jennings, 1976, for complications in the interpretation of cycloheximide effects on uptake systems).

\section{Kinetics}

The rate of uptake was measured over 5 min periods for wild-type cells in medium containing polyamine at concentrations between 0.35 and $5.0 \mathrm{mM}$, and $K_{\mathrm{m}}$ and $V_{\max }$ values were estimated using the direct linear plot method of Eisenthal \& Cornish-Bowden (1974). The results (Table 1) are fairly self-consistent, with the exception of one spermidine uptake experiment which showed low values for both $K_{\mathrm{m}}$ and $V_{\max }$.

\section{Competition}

Table 2 shows the results of competition experiments in which uptake of $0.1 \mathrm{~mm}$ labelled putrescine or spermidine was measured in the presence or absence of $4 \mathrm{~mm}$ unlabelled putrescine, spermidine or spermine. Neither spermidine nor spermine significantly inhibited the transport of putrescine, but spermidine uptake was effectively inhibited by putrescine and spermine. Moreover, the affinity of the spermidine transport system for the other two polyamines appeared to be similar to that for spermidine.

\section{Regulation}

The transport of polyamines was studied in wild-type cells grown under various conditions for $3 \mathrm{~h}$ after the initial period of growth ( $17 \mathrm{~h}$ with $1 \%$ glucose and $10 \mathrm{~mm}$-nitrate), in an attempt to elucidate its regulation (Table 3 ). The highest activities were obtained after nitrogen starvation, when the rate of uptake for putrescine was approximately $2 \frac{1}{2}$ times faster 
Table 3. Uptake of polyamines after various treatments

Mycelium of the wild-type (biAI) strain was grown for $17 \mathrm{~h}$ on NLM $+10 \mathrm{~mm}$-nitrate and then shifted to the treatment medium indicated for a further $3 \mathrm{~h}$ before measurement of uptake of ${ }^{14} \mathrm{C}$-labelled polyamines from $3 \mathrm{~mm}$ solutions.

Treatment medium

NLM (nitrogen-free)

NLM - glucose (nitrogen- and carbon-free)

$\mathrm{NLM}+5 \mathrm{mM}$-alanine

$\mathrm{NLM}+2 \cdot 5 \mathrm{mM}$-urea

$\mathrm{NLM}+5 \mathrm{mM}$-nitrate

$\mathrm{NLM}+5$ mM-ammonium

$\mathrm{NLM}+10 \mathrm{mM}$-ammonium

\begin{tabular}{ccc}
\multicolumn{3}{c}{ Uptake $\left[\mathrm{nmol} \mathrm{min}^{-1}(\mathrm{mg} \text { wet wt })^{-1}\right]$} \\
\hline Putrescine & Spermidine & Spermine \\
0.39 & 0.17 & 0.11 \\
0.13 & 0.055 & 0.038 \\
0.22 & 0.071 & 0.076 \\
0.22 & 0.076 & 0.083 \\
0.10 & 0.060 & 0.045 \\
0.053 & 0.026 & 0.066 \\
0.040 & 0.023 & 0.050
\end{tabular}

\section{Table 4. Uptake of polyamines by the wild-type (biA1) and mutant strains}

Mycelium was treated for $3 \mathrm{~h}$ in nitrogen-free medium before measurement of uptake of ${ }^{14} \mathrm{C}$-labelled polyamines from $3 \mathrm{~mm}$ solutions.

\begin{tabular}{|c|c|c|c|}
\hline \multirow[b]{2}{*}{ Strain } & \multicolumn{3}{|c|}{ Uptake $\left.\left[\text { nmol } \min ^{-1} \text { (mg wet wt }\right)^{-1}\right]$} \\
\hline & Putrescine & Spermidine & Spermine \\
\hline biAl & 0.39 & $0 \cdot 16$ & 0.12 \\
\hline biAl; puA2 & 0.42 & $0 \cdot 16$ & $0 \cdot 12$ \\
\hline biA1; areA'550 & 0.025 & 0.016 & 0.040 \\
\hline
\end{tabular}

than that for spermidine, which in turn was $1 \frac{1}{2}$ times faster than that for spermine. Treatment for $3 \mathrm{~h}$ with alanine or urea as nitrogen sources or starvation for both carbon and nitrogen resulted in reduced uptake of all three polyamines. The most significant reduction occurred after treatment with nitrate or ammonium, which suggests that the system is nitrogen repressible. In a separate experiment, addition of $10 \mathrm{~mm}$-ammonium to the NLM uptake medium of nitrogen-starved cultures caused approximately $40 \%$ inhibition of spermidine uptake. The reduction in uptake produced by growth on ammonium (Table 3) was $85 \%$, so it appears likely that, as with glutamate uptake (Pateman et al., 1974), ammonium both inhibits uptake in the short term, and in the longer term represses synthesis of the uptake system. As would be expected for such a system the hyper-repressed allele of the ammonium repression are $A$ gene (Pateman \& Kinghorn, 1976, 1977) confers very low uptake activity (Table 4).

\section{DIS CUSSION}

Sneath (1955) found that the putrescine auxotrophs of $A$. nidulans would grow on $0.06 \mathrm{~mm}$-putrescine, but although conversion on an equimolar basis to spermidine is the only known function of putrescine, Sneath found that spermidine was only one-tenth as active as a supplement for the mutant. Similarly, Hope \& Stevens (1976) found that if putrescine was replaced by spermidine, a 100 -fold greater concentration was required to maintain similar growth rates. They suggested that this could be explained by their finding of a 40 -fold difference in uptake of the two polyamines from dilute solutions over a period of $9 \mathrm{~h}$. Here we have confirmed that both putrescine and spermidine are actively concentrated from very dilute solutions and have attempted to characterize the uptake systems by measurements of uptake over $5 \mathrm{~min}$ periods during which it is hoped that uptake should not be seriously complicated by subsequent metabolism. 
It is clear that in all experiments where putrescine and spermidine uptake is compared for mycelium from the same culture (see Fig. 2, Tables 3 and 4) putrescine is taken up more rapidly than spermidine, but only by a factor of two or three. (A greater difference is apparent in Table 2, but here separate cultures were used for each experiment.) Attempts to describe the kinetics of polyamine transport (Table 1) agree with this conclusion and suggest that the difference lies mainly in the substrate affinities of the uptake systems, although the experiments were subject to inconsistencies not uncommon in transport experiments (cf. Tabor \& Tabor, 1966).

Putrescine and spermidine appear to be transported by independent systems (Table 2), and since putrescine uptake is not inhibited by spermidine, it seems unlikely that any significant amount of putrescine is transported by the spermidine system, although it does inhibit that system.

It is possible to calculate whether the observed polyamine transport would be expected to support the growth of the putrescine auxotroph. Bushell \& Bull (1974) found that cellular polyamine concentrations increased from 7 to $34 \mu \mathrm{mol}(\mathrm{g} \text { dry wt })^{-1}$ with increasing specific growth rate from 0.05 to $0.15 \mathrm{~h}^{-1}$. From this it can be calculated that at the fastest growth rate the kinetics of uptake shown in Table 1 should allow the necessary polyamines to be taken up from the medium if it contained $0.027 \mathrm{mM}$-putrescine, which compares quite well with the $0.06 \mathrm{~mm}$ concentration usually supplied. A fourfold greater concentration of spermidine should also suffice if these figures are taken at their face value.

There are three possible reasons why the uptake experiments reported here fail to reflect very closely the ability of the putrescine mutant to grow on exogenous polyamines. First, the relatively low accumulation of spermidine, and more strikingly spermine, over $30 \mathrm{~min}$ as opposed to $5 \mathrm{~min}$ intervals may depend on the inhibition of the relevant uptake systems by the accumulated polyamines. If the spermidine uptake system is inhibited by internal spermidine and spermine, as it is by these compounds when presented externally, uptake of these polyamines may decline rapidly as accumulation progresses. On the other hand, putrescine is not accumulated as such, but is rapidly converted to spermidine and spermine, as found in the $30 \mathrm{~min}$ accumulation experiment and elsewhere (Winther \& Stevens 1976), and these higher polyamines do not, at least when presented externally, inhibit further putrescine uptake.

Secondly, exogenous spermidine may go into an internal pool where it is less available to the cell than spermidine synthesized from putrescine. This would, however, be the reverse of the case for basic amino acids in Neurospora, where exogenous arginine in the cytosol is more available for metabolism than endogenous material which is stored in the vacuole (Bowman \& Davis, 1977). For polyamines, the nucleus may be a more important cellular compartment than the vacuole, but the concentration of spermidine within it is uncertain (Stevens \& Winther, 1979) and little is known about transport systems into the nucleus.

Thirdly, Winther \& Stevens (1976) found that polyamines are synthesized at much higher rates during conidial germination than in mature mycelium, probably because the specific growth rate is also very high during germination (Trinci, 1969). It is not easy to measure transport into very young cultures, but Kurtz \& Champe (1979) have shown that uptake of a number of substances was very much higher in $10 \mathrm{~h}$ than in $20 \mathrm{~h}$ cultures. The behaviour of the putrescine mutant could be explained if the transport systems for putrescine and spermidine are not magnified to the same extent in spore germlings when polyamine requirement is greatest. This is now being investigated further.

The 40-fold greater accumulation of putrescine compared with spermidine found by Hope $\&$ Stevens (1976) could result from the much smaller uptake differences reported here if the very low putrescine concentration $(0.43 \mu \mathrm{M})$ which they used was sufficient to stimulate some growth of the puAl strain over the $9 \mathrm{~h}$ accumulation period, while the same concentration of spermidine was below the threshold for growth stimulation.

Putrescine can act as a sole nitrogen source for $A$. nidulans (Spathas, 1978) so it is not unnatural that its uptake, like that of other complex nitrogen sources (Pateman \& Kinghorn, 
1977), should be repressed by ammonium. The repression by nitrate may be explained either by conversion to ammonium or by a direct action on nitrogen catabolism as proposed by Cove (1976). Spermidine, on the other hand, cannot act as a sole nitrogen source for $A$. nidulans, so the nitrogen regulation of its uptake may indicate that the uptake system is shared with other nitrogenous materials.

Studies are now continuing with further characterization of the spermidine uptake system, and in particular investigating the properties of a spermidine-sensitive mutant in which putrescine can be replaced by spermidine at much lower concentrations than in the wild-type.

We are grateful for the excellent technical assistance of Flora Kirkpatrick and wish to acknowledge the financial assistance to D.H.S. of the Ministry of Coordination and Planning of Greece.

\section{REFERENCES}

BaChrach, U. B. (1973). Function of Naturally Occurring Polyamines. New York: Academic Press.

Bowman, B. J. \& Davis, R. H. (1977). Cellular distribution of ornithine in Neurospora: anabolic and catabolic steady states. Journal of Bacteriology 130 , 274-284.

Bushell, M. E. \& Bull, A. T. (1974). Polyamine, magnesium and ribonucleic acid levels in steadystate cultures of the mould Aspergillus nidulans. Journal of General Microbiology 81, 271-273.

Cove, D. J. (1966). The induction and repression of nitrate reductase in the fungus Aspergillus nidulans. Biochimica et biophysica acta 113, $51-56$.

Cove, D. J. (1976). Chlorate toxicity in Aspergillus nidulans. Molecular and General Genetics 146, $147-159$.

Dion, A. S. \& Herbst, E. J. (1970). Polyamine changes during development of Drosophila melanogaster. Annals of the New York Academy of Sciences 171, 723-734.

Eisenthal, R. \& CoRnish-Bowden, A. (1974). The direct linear plot: a new graphical procedure for estimating enzyme kinetic parameters. Biochemical Journal 139, 715-720.

Hope, J. A. \& STEvens, L. (1976). The effects of putrescine, spermidine and spermine on the growth of a polyamine-requiring mutant of Aspergillus nidulans. Biochemical Society Transactions 4, $1128-1130$.

KuRTZ, M. B. \& Champe, S. P. (1979). Genetic control of transport loss during development of Aspergillus nidulans. Developmental Biology 70, 82-88.

JenNings, D. H. (1976). Transport and translocation in filamentous fungi. In The Filamentous Fungi, vol. 2, Biosynthesis and Metabolism, pp. 32-64. Edited by J. E. Smith \& D. R. Berry. London: Edward Arnold.

Pateman, J. A. \& Kinghorn, J. R. (1976). Nitrogen metabolism. In The Filamentous Fungi, vol. 2,
Biosynthesis and Metabolism, pp. 159-238. Edited by J. E. Smith \& D. R. Berry. London: Edward Arnold.

Pateman, J. A. \& Kinghorn, J. R. (1977). Genetic regulation of nitrogen metabolism. In Genetics and Physiology of Aspergillus, pp. 203-241. Edited by J. E. Smith \& J. A. Pateman. London: Academic Press.

Pateman, J. A., Kinghorn, J. R. \& Dunn, E. (1974). Regulation aspects of L-glutamate transport in Aspergillus nidulans. Journal of Bacteriology 119, $534-542$.

Pontecorvo, G., Roper, J. A., Hemmons, L. M., Macdonald, K. D. \& Bufton, A. W. J. (1953). The genetics of Aspergillus nidulans. Advances in Genetics 5, 141-238.

SNEATH, P. H. A. (1955). Putrescine as an essential growth factor for a mutant of Aspergillus nidulans. Nature, London 175, 818.

Spathas, D. H. (1978). Studies of a position effect and amine metabolism in Aspergillus. Ph.D. thesis, University of Glasgow, U.K.

Stevens, L. \& Winther, M. D. (1979). Spermine, spermidine and putrescine in fungal development. Advances in Microbial Physiology 19, 63-148.

TABOR, C. W. \& TABOR, H. (1966). Transport systems for 1,4-diaminobutane, spermidine and spermine in Escherichia coli. Journal of Biological Chemistry 241, 3714-3723.

TABOR, C. W. \& TABOR, H. (1976). 1,4Diaminobutane (putrescine), spermidine and spermine. Annual Review of Biochemistry 45, 285-306.

TrINCI, A. P. J. (1969). A kinetic study of the growth of Aspergillus nidulans and other fungi. Journal of General Microbiology 57, 11-24.

Winther, M. \& Stevens, L. (1976). Synthesis of polyamines in germinating conidia of Aspergillus nidulans. Biochemical Society Transactions 4, 1126-1128. 